\title{
PROGRAMA SAÚDE DA FAMÍLIA: ENTREVISTA ${ }^{1}$
}

\author{
DENISE. Porque o Ministério da Saúde (M.S.) propôs o Programa Saúde da Família \\ (PSF)? Fale do histórico do surgimento da proposta.
}

HELOIZA. Inicialmente é preciso afirmar que hoje o Programa Saúde da Família é uma das grandes prioridades do Ministério da Saúde, mas que não foi assim desde a sua origem. Em segundo lugar dizer que esta não é uma proposta nova. Estamos recuperando uma série de iniciativas e de movimentos inerentes ao próprio "Movimento Sanitário" no sentido de reordenamento do modelo assistencial em saúde e de consolidação do Sistema Único de Saúde. Em 1991 o Ministério da Saúde lançou o Programa de Agentes Comunitários de Saúde (PACS), com o objetivo de contribuir para o enfrentamento dos alarmantes indicadores de morbimortalidade infantil e materna na região Nordeste do Brasil. Este programa já estava pautado em princípios indutores da mudança de modelo, ou seja, provocava a vinculação dos indivíduos e famílias com as unidades básicas de saúde. $O$ agente comunitário representava o "elo" entre a comunidade e o serviço de saúde. Este agente, sendo uma pessoa da própria comunidade e portanto identificado com seus valores e costumes, passou a contribuir significativamente no resgate e na valorização do saber popular.

Penso que também é importante citar as experiências brasileiras que, influenciadas ou não pelas recomendações de Alma Ata, já desenvolviam trabalhos importantes nesta direção e influenciaram o desenho e concepção do Programa de Agentes Comunitários de Saúde. Projetos como do Vale do Ribeira em São Paulo, Casa Amarela em Recife, Rondonópolis no Mato Grosso e especialmente o programa do Governo Estadual do Ceará foram importantes neste contexto. Também não posso deixar de citar que existiam inúmeras experiências, como a nossa em Santa Catarina (Projeto Costeira do Pirajubaé), que não fizeram parte do "desenho" do programa nacional, mas contribuíram para disseminar idéias e formar recursos humanos neste movimento de busca de alternativas que pudessem provocar mudanças no modelo assistencial.

No entanto, mesmo com todas as suas potencialidades, este programa percorria um caminho marginal no próprio espaço institucional, e foi avaliado externamente com muitos preconceitos. No nível de execução municipal, havia um limitante importante para a continuidade e o impacto das suas ações: as unidades básicas de saúde continuavam atuando no sistema tradicional, sem absolutamente nenhuma vinculação com a população, com baixa resolutividade e com um enfoque predominante curativo. Em outras palavras, atuando em direção oposta ao que se preconizava com o trabalho dos agentes comunitários.

Na medida que o PACS se fortalecia nos municípios, demonstrando resultados concretos nos indicadores de mortalidade infantil e sendo bem avaliado pela própria população, estas contradições cresciam e passaram a pressionar as unidades básicas de saúde. É importante citar que, além do impacto nos indicadores, o PACS trazia contribuições importantes para a organização dos sistemas locais através de uma expressiva interiorização de profissionais enfermeiros e do estímulo à criação dos conselhos municipais de saúde (eram exigências para a implantação do programa).

Alguns municípios, mais engajados no processo de fortalecimento do sistema de saúde e mais articulados tecnicamente, passaram a fortalecer este debate. Expandiu-se então a idéia de que o Ministério da Saúde precisava ampliar os investimentos no sentido de dar retaguarda a este trabalho, uma vez que a idéia de "elo de ligação" não mostrava-se tão verdadeira, uma vez que as unidades básicas de referência continuavam desestruturadas, sucateadas e sem vínculo nenhum com a população.

Na continuidade deste processo, o Ministério da Saúde lança, no início de 1994, o Programa de Saúde da Família, que valorizava os princípios de territorialização, de vinculação com a população, de garantia de integralidade na atenção, de trabalho em equipe com enfoque multidisciplinar, de ênfase na promoção da saúde com fortalecimetno das ações intersetoriais e de estímulo à participação da

${ }^{1}$ Entrevista realizada por Denise Elvira Pires de Pires, membro do Conselho Editorial da REBEn com Heloiza Machado de Souza, diretora do Departamento de Atenção Básica do Ministério da Saúde. 
comunidade, entre outros.

Esta questão não pode ser entendida de forma descontextualizada. Naquele período a descentralização e a municipalização dos serviços de saúde estavam no foco do debate, como grande desafio para a implantação efetiva do Sistema Único de Saúde (SUS). Desta forma a adesão de secretários municipais de saúde foi expressiva e significante para o direcionamento da proposta do PSF - ele nasce num contexto de movimento dos próprios municípios. Porém não havia no MS, naquele momento, uma postura política forte o suficiente para definir o Saúde da Família como a estratégia de reordenamento do modelo assistencial.

O Programa nasce mesmo como algo marginal, tanto assim que se origina como um programa de um Departamento de Operações da Fundação Nacional de Saúde, sem nenhuma articulação com os outros setores do Ministério da Saúde.

Não havia, no momento do seu lançamento, uma definição de orçamento que garantisse sua expansão. A modalidade de financiamento do sistema de saúde também era completamente contraditória com os princípios do PSF. Propunha-se no PSF a "produção social da saúde" com um modelo de financiamento que remunerava procedimentos executados, em detrimento de todas as ações de prevenção de doenças e de promoção da saúde.

Não se pautou no MS, naquele momento, o desenvolvimento de uma política de recursos humanos que pudesse fomentar ou recuperar profissionais com perfil para este modelo.

Desta forma fica claro que o PSF nasce em condição completamente desfavorável. A questão de recursos humanos, aliada ao financiamento, eram apontados como os maiores "nós" críticos para a sustentabilidade da proposta.

Não tenho dúvida de que houve muita persistência técnica naquele momento. A ousadia de alguns secretários municipais foi fundamental para mostrar que o caminho era possível, que o PSF representava um potencial importante para reordenar a atenção básica e provocar a reorganização dos demais níveis de complexidade do sistema. Desta forma não estava se caracterizando como "paralelo ao SUS" e sim como estratégia de substituição das práticas tradicionais da atenção básica.

O primeiro documento do PSF falava em focalizar os 32 milhões de excluídos, segundo o mapa da fome do IPEA (Instituto de Pesquisas Econômicas Aplicadas). Isso também gerou a compreensão equivocada de que o PSF era um "programa de pobre para pobre". Diante da limitação de recursos, optou-se por iniciar a implantação do programa pelas áreas mais desassistidas, de grande exclusão social, enquanto compromisso com o princípio da equidade.

A partir de 1995 o PSF passou a ser incorporado pela Secretaria de Assistência à Saúde e iniciou uma trajetória importante para sair da condição "periférica" no MS e que o levou a ocupar hoje o espaço de prioridade, não somente do Ministério da Saúde, mas configurando-se como um certo consenso entre os níveis estadual e municipal de gestão do sistema. Nesta trajetória o PSF gerou a organização do Departamento de Atenção Básica - hoje vinculado à Secretaria de Políticas de Saúde, que tem como missão o fortalecimento da atenção básica, tendo o PSF como seu eixo estruturante.

É no campo do financiamento que encontramos uma das mais significativas sinalizações de como se traduziu a prioridade dada a esta estratégia. Em 1994 o financiamento, como afirmei anteriormente, era extremamente frágil e inadequado. Hoje podemos não ter atingido o ideal, mas conseguimos avanços importantes a partir da operacionalização da NOB-SUS/96 (Norma Operacional Básica do Sistema Único de Saúde de 1996) com a implantação do PAB - Piso de Atenção Básica. O PAB introduz a modalidade per capita, com transferência regular e automática (do Fundo Nacional de Saúde aos Fundos Municipais de Saúde) dos recursos federais para os municípios habilitados como gestores da atenção básica (atualmente cerca de 98\% dos municípios já estão habilitados nesta condição de gestão), gerando maior autonomia para a execução das ações e atividades da atenção básica consideradas de responsabilidade típica municipal.

O PAB é composto por uma parte fixa (varia de 10 a 18 reais/habitante/ano) e uma variável destinada aos incentivos voltados a programas, estratégias ou ações prioritárias para o processo de reorganização da atenção básica. Referem-se aos incentivos de Agentes Comunitários de Saúde e Equipes de Saúde da Família, de combate às carências nutricionais, de epidemiologia e controle de doenças, às ações básicas de vigilância sanitária e assistência farmacêutica básica.A implantação 
Entrevista com a Diretora do Departamento...

do $P A B$, além do avanço enquanto modalidade que rompeu com a lógica de remuneração de procedimentos, também representou um caráter redistributivo importante no campo dos recursos financeiros. Para se ter uma idéia, antes da sua implantação, 1.943 municípios faturavam , em média, 3,3 reais per capita/ano nas ações básicas. Por outro lado, 269 municípios faturavam, em média, 22 reais/per capita/ano. A diferença entre o Estado que tinha o maior per capita e o que tinha o menor per capita era de $133 \%$. Esta diferença agora é de $25 \%$.

O incentivo ao Programa de Saúde da Família e de Agentes Comunitários também teve uma evolução importante. No início de 1998 o valor do incentivo era de 1.800 reais / agente comunitário / ano e o das equipes de saúde da família variava de 14 a 28 mil / equipe / ano. Em 1999, já na gestão do Ministro Serra, esse valor foi alterado para 2.200 reais / agente / ano e as equipes passaram a ter um incentivo fixo de 28 mil / equipe /ano.

No final de 1999 ocorreu uma nova alteração nos valores e na forma de repasse dos incentivos para as equipes de saúde da família. Criou-se uma classificação de nove faixas de valores de incentivo, que vai de 28 mil reais /equipe / ano a 58 mil reais/equipe/ ano de acordo com a cobertura populacional do programa nos municípios. Esta foi uma decisão para induzir o aumento da cobertura, de forma que o PSF se constitua de fato como substitutivo das práticas tradicionais, tornando-se a porta de entrada nos sistemas locais de saúde.

Estas mudanças na lógica do financiamento, aliado a outras medidas técnicas e políticas, se refletem nos números de equipes e de agentes.

Em dezembro de 1997 haviam 54.934 agentes comunitários de saúde e 1.623 equipes de saúde da familia implantados. Hoje são 145 mil agentes que acompanham mais de 80 milhões de pessoas. $\mathrm{E}$ são 10.400 equipes implantadas em todo o país, que já vinculam mais de 30 milhões de pessoas. Agora estamos estimulando o avanço desta estratégia para os grandes centros urbanos.

Podemos afirmar que nesses anos de implantação do PSF, temos percorrido importantes reformulações na direção do aperfeiçoamento e das adaptações necessárias para que se coloque de fato como uma estratégia capaz de contribuir para o reordenamento do modelo de atenção, a partir da atenção básica.

$D E N I S E$. A realidade tem mostrado que os trabalhadores que atuam na equipe do PSF não têm a mesma relação trabalhista que os demais trabalhadores das unidades básicas. Eles são contratados por tempo determinado, com condições de trabalho também diferenciadas. Existe perspectiva de integrá-los à equipe? Há uma perspectiva de mudança na organização do serviço como um todo?

HELOIZA. É preciso diferenciar em alguns aspectos. Se queremos de fato provocar mudanças nas práticas de trabalho, é fundamental que todos os profissionais exerçam dedicação em tempo integral. Para isso é preciso haver remuneração adequada. As pesquisas têm nos mostrado que os salários de médicos e enfermeiros, ainda que reproduzindo as diferenças entre si, têm sido atraentes no mercado.

Quanto à relação trabalhista, é preciso reconhecer que existe hoje uma certa precarização das relações de trabalho em todo o Sistema Único de Saúde e, portanto, não é "privilégio" do PSF. O que ocorre é que o PSF, pelo destaque político e pelo avanço de sua implantação, coloca este problema na 'vitrine'. Estamos certos de que este é um problema que precisa ser superado se queremos a sustentabilidade do PSF.

Quanto às condições diferenciadas de trabalho, é preciso entender o que se quer dizer: defendemos unidades básicas que ofereçam condições para o desenvolvimento dos trabalhos em padrões ideais de qualidade. Precisamos pensar em unidades básicas com incorporação adequada de tecnologia que permita elevar o grau de resolutividade na assistência e os níveis de satisfação e segurança dos profissionais. No entanto esta ainda não é a realidade. As equipes de saúde da família, em sua maioria, estão desenvolvendo suas atividades em unidades precárias - as mesmas utilizadas pela rede tradicional. Nas pesquisas recentes, que realizamos, constatamos a insatisfação dos profissionais quanto as condições de espaço físico, de equipamentos, de acesso a medicamentos e 
apoio diagnóstico. Isso, na minha avaliação, é positivo enquanto movimento reinvidicatório dos profissionais. Demonstra que as equipes não estão acomodadas com a precariedade das instalações que herdamos nas unidades básicas de saúde. Acho que estamos, onde incluo especialmente os municípios, num esforço muito grande para recuperar as condições de trabalho das unidades básicas, buscando incorporação tecnológica para garantir os padrões de qualidade desejados. No ano passado o MS introduziu um incentivo financeiro fixo, pontual, por equipe implantada, para apoiar as questões de infra-estrutura. Estamos, junto com as Secretarias Estaduais, aperfeiçoando também os investimentos em medicamentos básicos.

Quanto à integração dos profissionais nas equipes, acho que é uma deficiência no entendimento da proposta. A equipe do PSF é a própria equipe da atenção básica. O que está ocorrendo é um período transitório, de adaptação da rede tradicional ao novo modelo. Algo que temos induzido e recomendado aos municípios é que procurem absorver os profissionais que eles já possuem na rede. Se já existe um centro de saúde estruturado, com uma equipe de médicos, enfermeiros e auxiliares trabalhando, é possível converter esses profissionais para o novo modelo e estabelecer um novo processo de trabalho na unidade de saúde. É possível fazer, a nível municipal, uma convocação interna, para saber quem são os profissionais que querem se dedicar a esta nova modalidade de trabalho. É necessário esclarecer que irão assumir novos compromissos, que precisarão de dedicação integral e garantir que terão complementação de salário. Porém, continuam tendo vínculo institucional como já tinham. Quando se esgota a possibilidade deste aproveitamento, faz-se então uma seleção externa, com uma nova contratação .

No entanto, temos alguns exemplos onde a decisão local foi diferente e não se absorveu os profissionais já existentes. Isto freqüentemente gera resistência nos profissionais, levando a uma maior dificuldade de integração do PSF à própria rede de serviços.

\section{DENISE. Continuando sobre a questão do financiamento e a forma de contratação de Recursos Humanos para o PSF, qual é a estratégia a ser adotada?}

HELOIZA. O financiamento para custear os serviços de saúde não muda em função da forma de contratação de Recursos Humanos. A mudança no financiamento que houve recentemente no Ministério da Saúde diz respeito ao aspecto da cobertura de equipes, como afirmei anteriormente. Ainda é necessário pensar na questão das grandes cidades, pensar as equipes enquanto aglomerados de equipes. Na medida em que um grande município fecha com o Programa de Saúde da Família um território significativo, enquanto cobertura populacional, ele já merece ser analisado de forma diferenciada. A modalidade de financiamento atual ainda não dá conta desse aspecto. Um grande município precisa fazer um esforço imenso para atingir a cobertura de $70 \%$ da população, para corresponder à maior faixa do valor do incentivo.

Quanto à forma de contratação, isso é sempre uma decisão municipal. O que o Ministério tem feito é um esforço, nos últimos anos, para identificar modalidades de contratação compatíveis com o perfil de trabalho e dos profissionais que integram as equipes, mas entendendo que a decisão final, a escolha, é sempre do gestor municipal. Muitos municípios já resolveram a questão da contratação através do concurso público, garantindo regime CLT aos profissionais. O problema maior está na contratação dos agentes comunitários, que exige um tratamento diferenciado em função do perfil desses trabalhadores.

Pensar em aplicar o concurso público para os agentes é abrir mão da condição de morador da própria comunidade, pois o concurso traz em si o caráter universalista no momento da inscrição dos candidatos. E perder essa vinculação do agente com a própria comunidade é romper com um dos princípios elementares desta estratégia. Por isso temos trabalhado no levantamento de outras alternativas de contratação que garantam os direitos trabalhistas do regime CLT para os Agentes Comunitários. É uma articulação que envolve várias instituições e representações, como dos próprios agentes, dos Conselhos de Secretários Estaduais e Municipais de Saúde, do Ministério Público do Trabalho, do Ministério da Justiça, do Programa Comunidade Solidária.

Uma alternativa identificada como compatível para a contratação dos agentes comunitários de 
saúde, e que foi acatada recentemente pelo ministério Público do Trabalho, é a elaboração de Termo de Parceira entre as administrações municipais e entidades reconhecidas como a OSCIP - Organização da Sociedade Civil de Interesse Público. As OSCIPs foram regulamentadas em 1999 pela Lei 9.790/99 (conhecida como a lei do Terceiro Setor).

Repito que não é o Ministério da Saúde que vai resolver este problema. A decisão é do município, uma vez que o mesmo tem autonomia para escolher a modalidade de contratação adequada à sua realidade. Porém, entendemos que cabe ao Ministério da Saúde a responsabilidade de orientar e de ofertar os instrumentos que possam facilitar a decisão dos municípios, assim como a operacionalização de sua decisão.

Neste momento há um ingrediente novo neste debate, que é a recente aprovação da Lei de Responsabilidade Fiscal, que tem aspectos que interferem na questão de expansão do quadro de recursos humanos dos municípios. Há um processo de discussão já instalado envolvendo os níveis federal, estadual e municipal de gestão do SUS para a busca de soluções aos problemas já identificados na implantação desta lei.

DENISE. Ainda sobre a estratégia de que falávamos anteriormente...É possível observar que existe uma disputa política muito grande no que diz respeito ao modelo assistencial. De um lado, o modelo que tem por base os princípios aprovados na $8^{\text {a }}$ Conferência Nacional de Saúde. De outro, o modelo defendido pela maioria da categoria médica e pelas empresas de medicamentos e equipamentos hospitalares, baseado na atenção curativa, individual e fragmentada em especialidades. São dois modelos polares e teoricamente diferentes. Então, neste cenário, o Ministério da Saúde, ao invés de propor uma mudança radical no modelo assistencial com base nos princípios do SUS, opta pela estratégia de criar um Programa?

HELOIZA. Inicialmente é preciso entender que o PSF não está em contraposição ao SUS. Muito ao contrário, o PSF é uma estratégia que está contribuindo para a operacionalização dos próprios princípios do SUS. É preciso refletir sobre os caminhos percorridos no processo de construção do SUS. Mesmo que ainda não tenhamos atingido os princípios do SUS em sua plenitude, é preciso reconhecer que estamos construindo um sistema solidário e que os avanços, nestes últimos 10 anos, foram significativos, especialmente no que diz respeito ao processo de descentralização e municipalização dos serviços de saúde. O acesso aos serviços também foi ampliado, decorrente da própria expansão dos serviços municipais de saúde. No campo do financiamento avançamos significativamente, hoje a maior parte dos recursos federais já são transferidos pela modalidade fundo a fundo e recentemente o setor obteve uma importante conquista com a aprovação da PEC da saúde. Mesmo que os Conselhos de Saúde ainda não estejam atuando no plano ideal, é inegável o que já representam enquanto instância de participação e controle social.

No campo dos arranjos institucionais, a criação das Comissões Bipartites e Tripartites significa espaço privilegiado de pactuação entre os diferentes níveis de gestão do SUS. Não se admite mais decisões centralizadas, que não sejam acordadas entre as representações de gestores. Estes são alguns aspectos, dentre muitos, que demonstram os avanços. Mas também temos a consciência de que em algumas questões avançamos muito pouco. Ainda não conseguimos traduzir efetivamente estes avanços em uma linguagem que a população realmente compreenda: de serviços que sejam verdadeiramente acessíveis, oportunos, resolutivos e humanizados. É neste contexto que o PSF precisa ser entendido. É preciso criticar as suas limitações, mas é preciso também romper com preconceitos e reconhecer a sua potencialidade como estratégia estruturante do próprio Sistema Único de Saúde.

Outra questão importante, de amadurecimento, é a compreensão de que os avanços e retrocessos no interior do SUS não são de responsabilidade única do Ministério da Saúde. Esta é uma construção de erros e acertos que precisa ser compartilhada entre os três níveis de gestão e com a representação da sociedade. Por isso, quando o Ministério coloca hoje o Programa Saúde da Família como a estratégia de reorientação do modelo, com ampla adesão de gestores estaduais e municipais 
de saúde, o que resulta é o que alguns autores tem denominado de "reforma da reforma", ou seja, o PSF está provocando um movimento de reorientação do modelo no interior do Sistema Único de Saúde. Neste contexto estamos tendo avanços sucessivos, mas as transformações não ocorreram repentinamente e simultaneamente, e nem serão assim num país com tanta diversidade como o nosso.

Estamos agora vivendo a possibilidade de um novo salto na organização do sistema. Os princípios de regionalização e hierarquização do SUS também foram muito bem desenhados, mas representaram avanços limitados. Tivemos, sem dúvida, processos muito bem desenvolvidos, limitados a alguns municípios em um processo claro de atomização. A organização da atenção básica é condição para que qualquer sistema se estruture de forma hierarquizada. Os avanços provocados pela implantação do PSF nos últimos anos contribuiram para fortalecer a necessidade de avançar na organização dos demais níveis de complexidade, inclusive possibilitando uma atenção básica com maior incorporação tecnológica e maior capacidade resolutiva. Esta é a grande discussão que ocupa os fóruns tripartites nos últimos meses. Há, nitidamente, uma coesão de interesses das representações federal, estaduais e municipais para se iniciar em 2001 a operacionalização da instalação de redes regionalizadas com microregiões de saúde.

Isto tudo é um processo e é democrático porque é constantemente pactuado.

Acho que estamos em um momento privilegiado enquanto possibilidade de avanços, de aperfeiçoamento do processo de descentralização e com um compromisso claro de buscar a equidade na organização do nosso sistema de saúde.

\section{$D E N I S E$. Que papel vai ter o agente comunitário de saúde, já que esta é uma questão polêmica?}

HELOIZA. Realmente esta é uma polêmica antiga e o perfil do agente comunitário precisa ser e está sendo alterado diante das diferentes realidades e contextos de trabalho. É preciso lembrar que o agente comunitário começou a ser implantado no Nordeste, com um volume de atribuições e competências muito voltado ao enfrentamento da mortalidade infantil e materna. Este papel se expandiu, na região Norte, para o enfrentamento do cólera. Esta agenda básica de trabalho na área da mulher e da criança ainda permanece porque ainda é um grave problema em muitos municípios do país.

Ainda convivemos com problemas antigos, nos quais os agentes estão trazendo uma grande contribuição, a exemplo da malária, da tuberculose e da hanseníase. Com as transformações que estamos vivendo no quadro demográfico e epidemiológico a agenda requer permanente adaptação. Temos as questões relativas às doenças crônico- degenerativas, ao envelhecimento da população, à gravidez precoce, a expansão que ocorreu com a AIDS no país.

Com a expansão do Programa para outras regiões do país e sua implantação nos grandes centros urbanos a agenda de trabalho se altera mais rapidamente. A realidade urbana traz novos desafios que requerem uma ação intersetorial mais efetiva: é o enfrentamento de situações geradas pelo consumo de álcool e drogas, da violência intrafamiliar, da inclusão da saúde mental como agenda básica de trabalho.

A entrada dos agentes comunitáíiús de saúde nas regiões metropolitanas rompeu a visão que muitos tinham de que o agente comunitário somente era importante e necessário para as regiões rurais ou para o Nordeste do país. A avaliação que hoje a coordenação do Projeto QUALIS / SAÚDE DA FAMÍLIA de São Paulo faz, é que a grande inovação do Saúde da Família na capital de São Paulo é justamente a introdução do agente comunitário, por esta identidade que ele tem com as pessoas, pela facilidade de interlocução, pela capacidade de facilitar o vínculo com o restante da equipe e pela possibilidade de traduzir os interesses, necessidades e potencialidades da própria comunidade. Temos que entender que o agente tem, em qualquer contexto, a atribuição importante de provocador da vinculação, o que possibilita a criação dos laços de compromisso e de co-responsabilidade entre os serviços, as equipes e a população. Por tudo isso, podemos pensar no papel que os agentes terão no futuro, mas ainda precisamos trabalhar muito para superar os problemas com os quais ainda convivemos no presente. 
$R E B E n$. Será que, a longo prazo, não poderá haver confusão na divisão de papéis, dentro da equipe de saúde? Como o agente comunitário se diferencia dos outros membros da equipe? Qual é a sua especificidade?

HELOIZA. A grande especificidade do ACS, que o diferencia da equipe, é que ele vive e intervém no processo saúde-doença da comunidade num nível de interação próprio de quem participa de um mesmo universo simbólico, de uma mesma cultura. Assim, embora todos estejam preocupados com questões de promoção à saúde, o ACS é o elemento central para fomentar movimentos sociais por melhores condições de vida (moradia, transporte, educação, lazer, acesso a serviços de saúde), pelo sentimento único de pertencer àquela comunidade. Nesta perspectiva, ele atende aos princípios da promoção à saúde definidos nas conferências internacionais, conforme expressão da própria Carta de Otawa: construção de políticas públicas saudáveis, criação de ambientes saudáveis, fortalecimento da participação comunitária , desenvolvimento de habilidades pessoais e reorganização dos serviços de saúde.

Acabamos de concluir um documento importante que trabalha as competências dos agentes comunitários, mediante as diversas possibilidades de sua inserção na comunidade. Com isto estamos propondo uma qualificação profissional, em nível básico, para o ACS, de acordo com a lei 93.94/96 (LDB) .

DENISE. Se o agente comunitário é um trabalhador de saúde e se ele está incluído na equipe, poderíamos falar então do surgimento de uma nova profissão na área de saúde?

HELOIZA. Tem um debate importante neste aspecto. Ele é um agente de saúde hoje, mas com a ampliação da sua agenda e com a necessidade de articulação intersetorial, visando o desenvolvimento local, será ele um futuro agente de desenvolvimento social? Será um agente de cidadania? Qual a relação que isto tem com o desenvolvimento local sustentável, integrado?

Embora na maioria dos municípios ainda não conseguimos dar conta de vencer os desafios da agenda básica, é importante pensar que o agente comunitário se apresenta como um ator importante neste processo mais ampliado, de fortalecimento de um "pensamento intersetorial".

\section{DENISE. Como este trabalhador da saúde pode ser representado por um sindicato?}

HELOIZA. Eles já se organizam. Temos informação da existência de oito Federações e uma Confederação de Agentes Comunitários de Saúde.

DENISE. Dentro do âmbito do Ministério da Saúde, de um lado temos o PROFAE que busca a profissionalização dos trabalhadores de enfermagem. De outro lado, o próprio Ministério da Saúde forma Agentes Comunitários de Saúde e tem colocado no mercado uma grande quantidade de trabalhadores que acabam assumindo o papel de atendente de enfermagem. Em que se apóia esta contradição?

HELOIZA. Acho que não podemos nos confundir com esta questão. Em primeiro lugar os agentes comunitários não estão assumindo o papel de atendentes de enfermagem. Em segundo lugar é fundamental, para a qualidade da assistência, o investimento que está sendo articulado pelo PROFAE. Representa o que a enfermagem busca há muito tempo.

Existem também perguntas como: "Por que não transformar os agentes em auxiliares de enfermagem?" Temos colocado que isto pode ocorrer quando representa uma demanda pessoal, do próprio agente. Se ele quiser se incorporar no serviço como um auxiliar de enfermagem, então que se ofereça a oportunidade para que ele faça isto dentro dos processos de qualificação já existentes. 


\section{DENISE. Embora o PROFAE exclua o agente de saúde de seu programa...}

HELOIZA. Ele entra se for uma demanda do serviço, para que ele possa exercer o papel de auxiliar na Unidade. Um processo de transformação dos agentes em auxiliares de enfermagem não é o objetivo. O PROFAE é um programa que alocou recursos para qualificação profissional dos atendentes de enfermagem, isto é, qualificar, e também requalificar os auxiliares de enfermagem. A forma como o projeto foi estruturado e vem sendo gerenciado é que permite a criação de espaços para discutir a formação dos trabalhadores de nível médio para saúde. A qualificação profissional dos ACS é uma outra proposta que prevê a qualificação de nível básico, cuja diretrizes foram elaboradas com a finalidade de qualificar o ACS de acordo com os pressupostos da própria LDB.

\section{$D E N I S E$. E em relação a capacitação de recursos humanos para atuar no PSF, quais são os desafios?}

HELOIZA. A questão de recursos humanos representa desafios e riscos para a sustentabilidade deste modelo. Desde que o PSF foi concebido sabia-se da inexistência de profissionais com o perfil necessário. Sabia-se também que as instituições de ensino superior só ingressariam em um movimento de preparar profissionais com este perfil se houvesse mercado de trabalho que os absorvesse. $D$ e s $t$ a forma é preciso compreender que, durante uma longa transição, as equipes de Saúde da Família não serão compostas por profissionais com perfil ideal. Como em outros países, o Brasil também terá um processo de adaptação dos profissionais que já estão no mercado de trabalho para este novo modelo de atenção. A meta do Ministério da Saúde, com recursos previstos no PPA - Plano Plurianual do Governo Federal, aponta para a implantação de 20 mil equipes de saúde da família até o ano 2002. Isso representará a cobertura de praticamente metade da população brasileira. Creio que isto deve representar estímulo e uma certa segurança às escolas médicas e de enfermagem, para começar a produzir mudanças na formação dos seus profissionais.

Na verdade, o PSF veio fomentar um movimento já existente de necessidade de reforma do ensino na área da saúde. É preciso que as escolas se voltem para a realidade social e sanitária do país. É preciso que se tenha a capacidade de recuperar os elos perdidos na relação dos profissionais de saúde com a população. Há um certo consenso, além do PSF, destas necessidades. O Ministério da Saúde vem estimulando a participação das instituições de ensino superior, especialmente cursos de enfermagem e de medicina, a envolverem-se neste processo através dos Pólos de Capacitação, Formação e Educação Permanente em Saúde da Família. Atualmente já temos mais de cem cursos envolvidos neste processo, sendo 64 deles, cursos de enfermagem.

Os Pólos estão atuando no sentido de preparar os profissionais que estão sendo inseridos nas equipes e também provocar a formação dos futuros profissionais que ingressarão neste mercado de trabalho. Estamos falando de grandes números. Somente em relação aos profissionais de nível superior estamos falando em 10.400 equipes, ou seja, 10.400 médicos(as), 10.400 enfermeiros(as) onde soma-se ainda cerca de 2.000 enfermeiros(as) que estão nas áreas do programa de Agentes Comunitários que ainda não foram incorporadas pelo PSF.

O PSF portanto, provoca uma mudança concreta de espaço de trabalho, de mercado. O papel das Universidades tem sido fundamental. Esta é uma tarefa que de fato precisa ser compartilhada entre as instituições de ensino e de serviço.

Para atuar numa equipe de saúde da família o profissional precisa compreender a nova dinâmica do processo de trabalho. É preciso portanto inserí-lo em uma capacitação introdutória. Os profissionais precisam desenvolver os conhecimentos, habilidades e atitudes necessárias a nova concepção de trabalho, de forma a estarem preparados ao enfrentamento de situações e problemas no cotidiano da comunidade que está sob sua responsabilidade. Para isso é indispensável um processo de educação permanente. Outro movimento está voltado para a formação dos novos profissionais, seja através de inovações na graduação ou na pós-graduação. 
Entrevista com a Diretora do Departamento...

\section{DENISE. Este processo também envolve a Residência e a Especialização em Saúde da Família, cujas propostas estão sendo aprovadas agora...}

HELOIZA. Exatamente isto. Induzimos esta proposta para estimular as Universidades a apostar no Programa e elevar a sua contribuição na formação dos profissionais necessários.

A Residência não vai dar conta do quantitativo de profissionais necessários, mas ela é importante no sentido de formação de massa crítica, de produção de quadros multiplicadores do processo de capacitação. A especialização, que deve incorporar o ensino presencial e à distância, representa uma possibilidade importante de aprimoramento dos conhecimentos e também de valorização dos profissionais.

DENISE. Estás afirmando que esta mudança no mercado realimenta a formação dos profissionais de saúde e que a formação de novos quadros também influencia o mercado?

HELOIZA. Não tenho dúvida em afirmar que a expansão e o fortalecimento do PSF tem exercido influência sobre as Universidades, que passam a reconhecer um novo espaço de trabalho e iniciam movimentos no sentido de dirigir, ainda que não totalmente, pelo menos alguns aspectos da formação para este campo de trabalho. A entrada da Universidade também influencia o mercado, que encontra na perspectiva da formação, possibilidade de sua expansão e também contribui para o aperfeiçoamento conceitual e teórico da estratégia. Podem as escolas de enfermagem negar o mercado de trabalho gerado pelo PACS e PSF? Pode o MS estar desatento da capacidade formadora e da distribuição territorial das escolas de enfermagem?

$\mathrm{Na}$ área da pesquisa e da avaliação, esta realimentação também tem sido fundamental. Nestes poucos anos de vigência do Programa, os processos de avaliação e monitoramento, têm fornecido subsídios importantes para repensar as coisas em nível nacional, estadual e municipal. O PSF também tem estimulado a elaboração de dissertações de Mestrado e teses de Doutorado nesta temática, o que tem sido muito bom para o aprofundamento conceitual.

\section{DENISE. Qual é a concepção teórica sobre família que embasa o Programa?}

HELOIZA. Entendemos a família como o espaço nuclear da agregação das pessoas, inseridas em um contexto social, devendo ser objeto prioritário e de focalização da atenção à saúde.

Existem centros acadêmicos aprofundando esta questão e estas contribuições serão bem vindas ao desenvolvimento dos trabalhos. As equipes poderão se beneficiar de um maior embasamento teórico para melhorar o entendimento de "que famílias são estas que estamos lidando?".

\section{quistados?}

DENISE. Como o Programa tem sido avaliado até agora? Que resultados foram con-

HELOIZA. A avaliação no setor saúde sempre é comprometida pela deficiência dos instrumentos de avaliação. No PSF estamos preocupados em acompanhar os resultados na organização dos serviços, na satisfação dos usuários e dos profissionais de saúde, na ampliação do acesso e no impacto nos indicadores de saúde. Estruturamos e implantamos um sistema de informação para acompanhamento da oferta de serviços pelas unidades básicas de saúde da família e de um conjunto de indicadores de resultados. Por este sistema será possível, a partir do próximo ano, um acompanhamento sistemático da evolução desses resultados. Na amostra existente já observa-se um melhora significativa nas taxas de mortalidade infantil, na elevação dos índices de aleitamento materno, na cobertura de pré-natal, no acompanhamento de hipertensos e diabéticos, entre outros. Entretanto, a maior importância deste sistema de informação está no município. Ele apresenta-se como uma ferramenta fundamental que possibilita ao gestor a tomada de decisões em tempo real para o enfrentamento dos problemas identificados. Algumas avaliações pontuais, de municípios, apontam para a influência da expansão da cobertura do PSF no processo de organização dos demais níveis de complexidade do 
sistema.

Existem também algumas pesquisas de caráter qualitativo sobre o nível de satisfação com o Programa em alguns municípios e estados. Ainda não temos um resultado disto em nível nacional. Os resultados mostram uma elevação no nível de satisfação das pessoas com o Programa; não só dos usuários, mas também dos profissionais de saúde, que enfatizam o resgate da humanização da assistência e do reconhecimento profissional. Então, apesar das condições de trabalho serem inadequadas porque os Postos de Saúde ainda têm condições muito rudimentares para o atendimento, e apesar da situação contratual dos profissionais também incomodar, o nível de satisfação é crescente. Todas as pesquisas mostram isso. Há uma pesquisa que o Ministério da Saúde encomendou e foi coordenada pela Professora Maria Helena Machado, da FIOCRUZ, que revela que a maioria dos profissionais tem uma visão otimista e de satisfação no seu trabalho. Mostra também que quase $90 \%$ dos médicos e enfermeiros consideram bom o entrosamento da equipe.

Neste momento está em curso uma pesquisa nacional para verificar as mudanças ocorridas no processo de organização dos sistemas locais de saúde após a implantação do PAB e um estudo de custos do PSF, em municípios selecionados.

\section{DENISE. A interdisciplinariedade parece ser algo fundamental não é mesmo?}

HELOIZA. Sem dúvida. Os profissionais das equipes de saúde da família necessitam de uma visão ampliada. É imprescindível ser resolutivo nas clínicas básicas, ser capaz de resolver pelo menos $80 \%$ dos problemas de saúde da população numa concepção de recuperação de condições adequadas de saúde e de qualidade de vida. Esta prática precisa estar pautada pelos princípios de promoção da saúde. Isto requer uma visão e uma ação ampliada, exigindo a incorporação de novos saberes e novos conhecimentos que tradicionalmente não foram incorporados na formação dos nossos profissionais de saúde. 